\title{
Laparoscopic Completion total Gastrectomy as an Alternative Procedure for Gastric Stump Cancer: A Case Control Study
}

Tohru Takahashi

Juntendo University Urayasu Hospital https://orcid.org/0000-0002-1130-0549

Noriyuki Inaki ( $\square$ n.inaki@viola.ocn.ne.jp)

Juntendo University Urayasu Hospital https://orcid.org/0000-0002-4241-5015

Hiroshi Saito

Ishikawa Kenritsu Chuo Byoin

Yusuke Sakimura

Ishikawa Kenritsu Chuo Byoin

Kengo Hayashi

Ishikawa Kenritsu Chuo Byoin

Toshikatsu Tsuji

Ishikawa Kenritsu Chuo Byoin

Daisuke Yamamoto

Ishikawa Kenritsu Chuo Byoin

Hirotaka Kitamura

Ishikawa Kenritsu Chuo Byoin

Shinichi Kadoya

Ishikawa Kenritsu Chuo Byoin

Hiroyuki Bando

Ishikawa Kenritsu Chuo Byoin

\section{Research article}

Keywords: Gastric Stump Cancer, Gastrectomy, Laparoscopy, Feasibility Studies

Posted Date: June 30th, 2020

DOl: https://doi.org/10.21203/rs.3.rs-37319/v1

License: (c) (1) This work is licensed under a Creative Commons Attribution 4.0 International License. Read Full License 


\section{Abstract}

Background

Complete gastrectomy for gastric stump cancer can be challenging due to severe adhesions; therefore, advanced techniques are required to perform laparoscopic surgery. This study aimed to evaluate the clinical outcomes of laparoscopic completion total gastrectomy for the treatment of gastric stump cancer.

\section{Methods}

Patient records from January 2010 to October 2018 were retrospectively evaluated. The patients were divided into two groups depending on whether they underwent open or laparoscopic gastrectomy. We compared patient characteristics; operative, clinical, and pathological data; survival rates; and prognosis between the groups.

\section{Results}

Twenty open and 17 laparoscopic completion total gastrectomies were performed. No significant differences in the distribution of the clinical T and $\mathrm{N}$ categories, clinical stage, and reconstruction methods of the initial gastrectomy between the two groups were observed. Laparoscopic gastrectomy resulted in a significantly longer operation time (230 vs. $182.5 \mathrm{~min} ; \mathrm{p}=0.026)$, lower blood loss ( $14 \mathrm{vs} .105 \mathrm{~mL} ; \mathrm{p}=0.0000179)$, and shorter period to the first flatus passage ( 2 vs. 3 days; $p=0.0000401)$ than open gastrectomy. No significant differences in the number of retrieved lymph nodes, duration of hospital stay, complication rate, and postoperative analgesic usage between the two groups were observed. No patients required conversion to open surgery in the laparoscopic-treatment group. Pathological findings revealed that the laparoscopic group had a smaller tumor size (not pathological $\mathrm{T}$ category) and less metastatic lymph nodes than the open group; this led to an earlier distribution of the pathological stage and better overall or disease-free survivals in the laparoscopic group.

\section{Conclusions}

Laparoscopic completion total gastrectomy was safely conducted without complications and mortality implicating the oncological validity for the treatment of gastric stump cancer. With sophistication of laparoscopic skills and advanced technologies, laparoscopic completion total gastrectomy may be the best way to perform less invasive surgery in terms of decreased blood loss and earlier recovery of intestinal peristalsis.

\section{Background}

The prognosis of gastric cancer after gastrectomy has improved, however, the incidence of cancer in the remnant stomach is increasing [1, 2]. Newly developed gastric cancer after partial gastrectomy for benign disease or gastric cancer is defined as remnant gastric cancer or gastric stump cancer (GSC), which is found in $1.1-6 \%$ of patients [2-6]. Complete resection of the carcinoma combined with a radical lymph node dissection is the only way to secure curability and improve the prognosis in patients who have no other complications [7]. Mesenteric lymph node metastasis around the gastrojejunostomy may worsen the prognosis of GSC [5]. A reported 5-year disease-specific survival rate for GSC was 7-20\% due to the advanced tumor stage [2]. However, recent reports have stated that the overall 5-year survival for GSC has improved to approximately $53-56 \%$, which remains at a lower level than that of proximal gastrectomy for primary gastric cancer (PGC) $[4,5,8-10]$. Therefore, the diagnosis of GSC at an early stage in patients who have undergone gastrectomy is important to reduce complications. However, complete gastrectomy for GSC has been difficult and invasive due to the severe adhesions that can occur fromthe previous procedures.

Laparoscopic gastrectomy has been confirmed to be safe with improved postoperative pain and earlier recovery than open gastrectomy [11-13]. However, only a few studies have reported the feasibility of laparoscopic completion total gastrectomy (LCTG) for GSC [14-21]. This study aimed to evaluate the feasibility, safety, and clinical outcomes of LCTG compared with those of open completion total gastrectomy (OCTG) to prove the oncological validity of LCTG.

\section{Methods}

\section{Study design and patient characteristics}

We performed a database search and identified 40 patients who had undergone surgery for GSC at Ishikawa Prefectural Central Hospital in Japan from January 2010 to December 2018. One patient who underwent bypass surgery, one patient who underwent staging laparoscopy, and one patient who underwent robot-assisted completion total gastrectomy were excluded from this study. The remaining 37 patients underwent gastrectomy for GSC and were further divided into two groups according to the initial approach of the operation: the LCTG group $(n=17)$ and OCTG group ( $n=20)$. The mean age of patients in the LCTG and OCTG groups was $71.9 \pm 8.1$ and $68.9 \pm 9.1$ years, respectively. The male-to-female ratios in the LCTG and OCTG groups were 12 to 5 and 16 to 4 , respectively. The medical records of all patients were retrospectively evaluated to compare the short-term surgical and long-term oncological outcomes. The patient characteristics are shown in Table 1. 
Table 1

Patient Characteristics

\begin{tabular}{|c|c|c|c|}
\hline & LCTG $(n=17)$ & OCTG $(n=20)$ & $P$ value \\
\hline Age (years: mean \pm SD) & $71.9 \pm 8.1$ & $68.9 \pm 9.1$ & 0.288 \\
\hline Gender (M : F) & $12: 5$ & $16: 4$ & 0.779 \\
\hline $\mathrm{BMI}\left(\mathrm{kg} / \mathrm{m}^{2}:\right.$ median$)$ & $20.49(15.5-28.2)$ & $20.63(16.7-28.0)$ & 0.821 \\
\hline Comorbidity & $3(0-4)$ & $1(0-4)$ & 0.378 \\
\hline Initial disease (Benign : Malignant) & $5: 12$ & $4: 16$ & 0.779 \\
\hline Time interval (years) & $11(1-56)$ & $15.5(2-56)$ & 0.385 \\
\hline Tumor location & & & 0.9699 \\
\hline Anastomotic site & 3 & 3 & \\
\hline Non anastomotic site & 12 & 14 & \\
\hline Sutured site & 1 & 1 & \\
\hline Whole stomach & 1 & 2 & \\
\hline cT stage $(1 a / 1 b / 2 / 3 / 4 a / 4 b)$ & $0 / 13 / 2 / 2 / 0 / 0$ & $1 / 7 / 5 / 3 / 1 / 3$ & 0.114 \\
\hline cN stage $(0 / 1 / 2)$ & $20 / 0 / 0$ & $17 / 1 / 2$ & 0.489 \\
\hline cStage(I/IIA/IIB/III/IVA/IVB) & $15 / 0 / 2 / 0 / 0 / 0$ & $12 / 1 / 2 / 2 / 2 / 1$ & 0.359 \\
\hline Initial approach (laparoscopy : open) & $6: 11$ & $3: 17$ & 0.294 \\
\hline \multicolumn{4}{|l|}{ LDG:LPG:LPPG:LSG:Others) } \\
\hline \multicolumn{4}{|l|}{ (BI:BIl:RY:E-G:G-G:others) } \\
\hline $\begin{array}{l}L C T G \text { laparoscopic completion total g } \\
\text { open proximal gastrectomy, OPPG op } \\
L P P G \text { laparoscopic pylorus preserving } \\
\text { esophagogastrostomy, } G-G \text { gastrogas }\end{array}$ & $\begin{array}{l}\text { letion total gastrect } \\
\text { omy, } L D G \text { laparoscc } \\
\text { oic segmental gastr }\end{array}$ & $\begin{array}{l}\text { nass index, } O D G \text { op } \\
\text { ctomy, LPG laparos } \\
\text { h I, Bll Billroth II, } R Y\end{array}$ & $\begin{array}{l}\text { ctomy, } O P G \\
\text { gastrectomy, }\end{array}$ \\
\hline
\end{tabular}

\section{Indication}

The use of LCTG for GSC was introduced to our institution in 2010. The exclusion criteria for LCTG are remarkable direct tumor invasion to other organs and patient conditions that preclude laparoscopy.

\section{Surgical Procedures}

A five-port surgical approach was used. The first 12-mm trocar was inserted at the umbilical area using the Hassan method. The other trocars were subsequently inserted carefully under laparoscopic viewing as in conventional laparoscopy [22]. A 10-mm, 30-degree oblique viewing laparoscope was used, and the $\mathrm{CO}_{2}$ pressure was maintained at $10-12 \mathrm{mmHg}$. The Harmonic Scalpel (Ethicon EndoSurgery Inc., Cincinnati, $\mathrm{OH}$ ), which is an ultrasonic-activated device, and the LigaSure (Medtronic, Minneapolis, MN) were used for adhesiolysis and radical lymphadenectomy. The extent of lymph node dissection was based on the recommendations for total gastrectomy from the Japanese gastric cancer treatment guidelines [23]. In cases of benign disease at the initial surgery, the intact gastric vessels were dissected from the root, and the resected specimen was extracted through the umbilical incision, which was enlarged to the minimum size required for extraction.

\section{Patients who underwent Billroth I reconstruction during the initial surgery}

The gastrosplenic ligament was resected, starting with the opening of the omental bursa, and adhesions between the posterior gastric wall and pancreas were carefully divided. The dissection was carefully performed because the inferior surface of the left lateral segment of the liver was often severely adhesive to the remnant gastric wall. Moreover, the pneumoperitoneum yielded bloodless dissection. After encirclement of the gastro-duodenal anastomosis, the duodenum was transected using a linear stapler. Next, the remaining dissection around the remnant stomach including the vessels was completed, and the lower esophagus was transected in the same manner.

\section{Patients who underwent Billroth II or Roux-en Y reconstruction during the initial surgery}

The afferent and efferent loops of the jejunum or Roux limb were resected, securing sufficient distance from the anastomosis. The mesenteric lymph nodes were dissected depending on the tumor size or invasion. 
Reconstruction after the complete removal of the GSC was performed with the Roux-en-Y method. The jejunum was transected $25 \mathrm{~cm}$ from the ligament of Treitz. Approximately $20 \mathrm{~cm}$ of the jejunum on the anal side was sacrificed, and the Roux limb was prepared. Jejunojejunostomy was performed with the $Y$ limb. The Roux limb was ascended through the antecolic route, and esophagojejunostomy was performed using the overlap procedure. The mesenteric gap at the $\mathrm{Y}$ limb and Petersen's mesenteric defect were closed by continuous suturing using barbed string.

\section{Statistical analysis}

Patient ages are presented as the mean \pm standard deviation, and all other values are expressed as the median with range. All statistical analyses were completed using R statistical software, version 3.5.0 (R Foundation for Statistical Computing, Vienna, Austria). Chi-squared, Fisher's exact, and Mann-Whitney $\mathrm{U}$ tests were performed for comparisons between the two groups. The cumulative 5-year survival rates were calculated according to the Kaplan-Meier method, and survival curves were compared using the log-rank test. The statistical significance level was set at $p<0.05$.

\section{Results}

No significant differences between the two groups in sex distribution, body mass index, and comorbidity incidence were observed. A total of 5 and 12 initial gastrectomies were performed in the LCTG group for benign and malignant diseases, respectively; a total of 4 and 16 initial gastrectomies were performed in the OCTG group for benign and malignant diseases, respectively. However, the difference observed between the groups was not statistically significant. The median time from the initial gastrectomy to the development of GSC was comparable between the LCTG and OCTG groups (11 vs 15.5 years, respectively; $p=$ 0.385). The most common tumor location in the LCTG and OCTG groups was the non-anastomotic site (12 [70.6\%] and 14 [70\%] cases, respectively). However, only three cases in each group had the tumor detected at the anastomotic site (17.6\% and $15 \%$ in the LCTG and OCTG groups, respectively).

Two (11.8\%) and seven (35\%) patients in the LCTG and OCTG groups, respectively, were diagnosed with tumor depth invasion greater than clinical T stage 3. However, no significant difference in the distribution of the clinical T stage between the groups was observed. No patients in the LCTG group had preoperative lymph node metastasis. Therefore, patients in the LCTG group, except for those with clinical T stage 3, were considered to be at clinical stage I. The clinical stages in the OCTG group were diversely distributed; however, no significant difference in the stage distribution between the groups was observed. In the initial gastrectomy, several patients in both groups were treated with laparoscopy. The type and reconstruction of the initial gastrectomies were diverse; however, no significant difference in the distributions for both groups was observed. The most common reconstruction method in the initial gastrectomy was Billroth I anastomosis in seven (41.2\%) and eight (40\%) patients in the LCTG and OCTG groups, respectively.

\section{Operative And Postoperative Short-term Outcomes}

The surgical outcomes are depicted in Table 2. The median operation time was 230 (140-260) min and 182.5 (130-217.5) min in the LCTG and OCTG groups, respectively, representing a significant difference $(p=0.026)$. The median estimated blood loss was significantly different between the two groups: 14 $(4-100) \mathrm{mL}$ in the LCTG group and $105(8-840) \mathrm{mL}$ in the OCTG group $(\mathrm{p}=0.0000179)$. No significant difference in the extent of lymphadenectomy and morbidity was observed between the two groups, although three patients in the OCTG group had major complications (Clavien-Dindo classification > grade IIla). No conversion to open surgery was reported in the LCTG group. The median time to first flatus passage was significantly shorter in the LCTG group than that in the OCTG group ( 2 vs. 3 days; $p=0.0000401)$. No significant difference between groups in analgesic usage after postoperative day 5 ( 0 vs. 1 time; $p=$ 0.334 ) or in the duration of postoperative hospitalization (13 vs. 14 days; $p=0.657$ ) was observed.

Table 2

Operative and Postoperative Short-term Outcomes

\begin{tabular}{|llll|}
\hline & LCTG $(\mathbf{n = 1 7 )}$ & OCTG $(\mathbf{n = 2 0})$ & $P$ value \\
\hline Operation time (min : median) & $230(140-400)$ & $182.5(130-360)$ & 0.026 \\
\hline Estimated blood loss (ml) & $14(4-100)$ & $105(5-840)$ & 0.0000179 \\
\hline Lymphadenectomy & & & 0.899 \\
\hline D0 : D1 : D1+ : D2 & $2: 10: 4: 1$ & $4: 11: 4: 1$ & 0.288 \\
\hline Complication (Clavien-Dindo) & 0 & 3 & 0.0000401 \\
\hline$<$ IIla : $\geq$ Illa & 0 & - & 0.334 \\
\hline Conversion to Open & 0 & $3(2-6)$ & 0.657 \\
\hline First Flatus passage (POD) & $2(1-3)$ & $1(0-19)$ & $14(9-271)$ \\
\hline Analgesic usage ( $\geq$ d5) & $0(0-14)$ & $13(9-26)$ & \\
\hline Postoperative hospital stay (days) & & \\
\hline LCTG laparoscopic completion total gastrectomy, OCTG open completion total gastrectomy, POD post operative days, $d 5$ day 5 \\
\hline
\end{tabular}

\section{Pathological Outcomes}


The pathological findings of the resected specimens are shown in Table 3. The median tumor size in the LCTG group was significantly smaller than that in the OCTG group ( 26 vs. $40 \mathrm{~mm} ; \mathrm{p}=0.0457)$. No significant difference between groups in the median number of retrieved lymph nodes $(11 \mathrm{vs.9.5;} p=0.437)$, depth of the tumor invasion $(p=0.12)$, extent of lymphatic metastasis $(p=0.0509)$, distant metastasis ratio $(p=1)$, and variation of histological type ( $p=1)$ were observed. However, the median number of metastatic lymph nodes was significantly lower in the LCTG group than that in the OCTG group (0 vs. 0.5; $p=$ 0.0108). Additionally, the pathological stage distribution in the LCTG group was lower than that in the OCTG group ( $p=0.0346)$.

Table 3

Pathological Outcomes

\begin{tabular}{|llll|}
\hline & LCTG $(\mathbf{n = 1 7 )}$ & OCTG $(\mathbf{n}=\mathbf{2 0})$ & Pvalue \\
\hline Tumor size $(\mathrm{mm})$ & $26(12-77)$ & $40(8-120)$ & 0.0457 \\
\hline Retrieved LN & $11(0-49)$ & $9.5(0-46)$ & 0.437 \\
\hline Metastatic LN & $0(0-2)$ & $0.5(0-9)$ & 0.0108 \\
\hline $\mathrm{pT}(1 \mathrm{a} / 1 \mathrm{~b} / 2 / 3 / 4 \mathrm{a} / 4 \mathrm{~b})$ & $3 / 9 / 2 / 3 / 0 / 0$ & $2 / 4 / 2 / 6 / 3 / 3$ & 0.12 \\
\hline $\mathrm{pN}(0 / 1 / 2 / 3 \mathrm{a})$ & $15 / 2 / 0 / 0$ & $10 / 4 / 4 / 2$ & 0.0509 \\
\hline $\mathrm{pM}(0 / 1)$ & $17 / 0$ & $19 / 1$ & 1 \\
\hline $\mathrm{pStage}(\mathrm{IA} / \mathrm{IB} / \mathrm{IIA} / \mathrm{IIB} / \mathrm{IIIA} / \mathrm{IIIB} / \mathrm{IIIC} / \mathrm{IV})$ & $12 / 2 / 1 / 2 / 0 / 0 / 0 / 0$ & $5 / 1 / 3 / 3 / 4 / 3 / 0 / 1$ & 0.0346 \\
\hline Histological type & & & 1 \\
\hline Differentiated : Undifferentiated & $9: 8$ & $11: 9$ & \\
\hline LCTG laparoscopic completion total gastrectomy, OCTG open completion total gastrectomy, LN lymph node \\
\hline
\end{tabular}

\section{Postoperative Long-term Outcomes}

The postoperative long-term outcomes are shown in Table 4. The median follow-up duration was 41 and 31 months for the LCTG and OCTG groups, respectively $(p=0.427)$. In the LCTG group, one patient died from recurrence, and one patient died from pneumonia. In the OCTG group, six patients died from recurrence, and three patients died from other diseases. Specifically, one patient in the LCTG group (5.88\%) and 11 patients in the OCTG group (55\%) developed recurrence, representing a significant difference $(p=0.004679)$. Recurrence in the patient in the LCTG group was due to metastasis to the mediastinal lymph nodes. In the OCTG group, seven cases of metastasis in the liver, three in the peritoneum, two in the pleura or regional lymph nodes, and one in the lung were reported. The 5 -year overall survival rate was significantly higher in the LCTG group than that in the OCTG group ( $84.4 \%$ vs. $48.5 \%$; $p=$ 0.0373) (Fig. 1). The 5-year disease-free survival of the LCTG group was significantly higher than that of the OCTG group $(93.3 \%$ vs. $41.9 \% ; p=0.00274)$ (Fig. 2).

Table 4

Postoperative Long-term Outcomes

\begin{tabular}{|c|c|c|c|}
\hline & LCTG $(n=17)$ & OCTG $(n=20)$ & $P$ value \\
\hline MST (month) & $41(0-99)$ & $31(4-80)$ & 0.427 \\
\hline Death & $2(11.8 \%)$ & $9(45.0 \%)$ & 0.06528 \\
\hline \multirow[t]{4}{*}{ Cause of death } & Original (1) & Original (6) & \\
\hline & Pneumonia (1) & Sepsis (1) & \\
\hline & & Pneumonia (1) & \\
\hline & & Malignant disease (1) & \\
\hline DFS (month: median) & $41(0-99)$ & $24(0-80)$ & 0.123 \\
\hline Recurrence & $1(5.88 \%)$ & $11(55.0 \%)$ & 0.004679 \\
\hline \multirow[t]{5}{*}{ Location } & LYM (1) & Liver (7) & \\
\hline & & Peritoneum (3) & \\
\hline & & Pleura (2) & \\
\hline & & LYM (2) & \\
\hline & & Lung (1) & \\
\hline
\end{tabular}


Table 5 Summary of Case Reports of LCTG for GSC

\begin{tabular}{|c|c|c|c|c|c|c|c|c|c|c|c|c|c|c|}
\hline Author & Year & $\mathrm{n}$ & age & $\begin{array}{c}\text { sex } \\
(\mathrm{M} / \mathrm{F}) \\
\end{array}$ & $\begin{array}{l}\text { initial } \\
\text { disease } \\
(\mathrm{B} / \mathrm{M})\end{array}$ & $\begin{array}{c}\text { previous } \\
\text { ope } \\
(\mathrm{O} / \mathrm{L}) \\
\end{array}$ & $\begin{array}{c}\text { previous } \\
\text { reconstr } \\
\text { uction }\end{array}$ & $\begin{array}{c}\text { interval } \\
\text { (yrs) }\end{array}$ & $\begin{array}{c}\text { Operation } \\
\text { time } \\
(\mathrm{min}) \\
\end{array}$ & $\begin{array}{c}\text { Blood } \\
\text { loss } \\
(\mathrm{ml}) \\
\end{array}$ & $\begin{array}{l}\text { open } \\
\text { CV } \\
(\mathrm{n}) \\
\end{array}$ & $\begin{array}{c}\text { Morbidity } \\
(\mathrm{C}-\mathrm{D} \text { III }>\text { ) } \\
(\mathrm{n})\end{array}$ & $\begin{array}{c}\text { Retrievec } \\
\text { LN } \\
(\mathrm{n})\end{array}$ & $\begin{array}{c}\text { Hospital } \\
\text { stay } \\
\text { (days) } \\
\end{array}$ \\
\hline Yamada H, et al. & 2005 & 1 & 69 & $1 / 0$ & $0 / 1$ & $1 / 0$ & BII & 10 & 274 & 30 & - & - & - & - \\
\hline Corcione F, et al. & 2008 & 3 & $59-73$ & $3 / 0$ & $3 / 0$ & $3 / 0$ & BII (3) & 15 & $\begin{array}{c}210 \\
(160-260)\end{array}$ & $\begin{array}{c}19 \\
(0-264)\end{array}$ & - & fistula (1) & $\begin{array}{c}18 \\
(12-26)\end{array}$ & $\begin{array}{c}11 \\
(8-18)\end{array}$ \\
\hline Cho HJ, et al. & 2009 & 2 & $37 / 68$ & $1 / 1$ & $0 / 1$ & $1 / 1$ & BII (2) & 1.5 & $\begin{array}{c}487.5 \\
(435-540)\end{array}$ & $\begin{array}{c}425 \\
(400-450)\end{array}$ & - & - & $\begin{array}{c}14.5 \\
(9-20)\end{array}$ & - \\
\hline Qian F, et al. & 2010 & 15 & $\begin{array}{c}53.5 \\
(40-76)\end{array}$ & $13 / 2$ & - & - & $\begin{array}{c}\text { BI (2) } \\
\text { BII (13) }\end{array}$ & - & $205 \pm 25$ & $110 \pm 40$ & 1 & SSI (1) & $18 \pm 5$ & - \\
\hline Shinohara T, et al. & 2013 & 5 & $\begin{array}{c}70.4 \\
(54-84)\end{array}$ & $3 / 2$ & $1 / 4$ & - & $\begin{array}{c}\text { BI (3) } \\
\text { BII (2) }\end{array}$ & - & $\begin{array}{c}370.8 \\
(258-540)\end{array}$ & $\begin{array}{c}63.6 \\
(10-233)\end{array}$ & - & - & $\begin{array}{c}18.2 \\
(12-24)\end{array}$ & $\begin{array}{c}8.8 \\
(8-9)\end{array}$ \\
\hline Pan et al. & 2014 & 3 & $\begin{array}{c}68.7 \\
(55-76)\end{array}$ & $3 / 0$ & $2 / 1$ & $3 / 0$ & $\begin{array}{l}\text { BII (2) } \\
\text { RY (1) }\end{array}$ & $\begin{array}{c}22 \\
(6-30)\end{array}$ & $\begin{array}{c}251.7 \\
(225-280)\end{array}$ & $\begin{array}{c}76.7 \\
(50-100)\end{array}$ & - & - & $\begin{array}{c}16.7 \\
(10-22)\end{array}$ & $\begin{array}{c}8 \\
(7-9)\end{array}$ \\
\hline Kim HI, et al. & 2015 & 1 & 83 & $0 / 1$ & $0 / 1$ & $1 / 0$ & BII & 28 & 200 & 100 & - & - & 24 & 13 \\
\hline Korehisa S, et al. & 2015 & 4 & $\begin{array}{c}76.5 \\
(62-83)\end{array}$ & $4 / 0$ & $1 / 3$ & $3 / 1$ & $\begin{array}{c}\text { BI (2) } \\
\text { BII (1) } \\
\text { RY (1) }\end{array}$ & $\begin{array}{c}19 \\
(0.1-50)\end{array}$ & $\begin{array}{c}413.3 \\
(367-488)\end{array}$ & $\begin{array}{c}270.3 \\
(33-500)\end{array}$ & - & $\begin{array}{l}\text { bleeding } \\
\text { (1) }\end{array}$ & - & $\begin{array}{c}18 \\
(13-26)\end{array}$ \\
\hline Kim DJ, et al. & 2016 & 1 & 73 & $1 / 0$ & $0 / 1^{*}$ & $1 / 0$ & GJ & 25 & 295 & 200 & - & - & 20 & 7 \\
\hline Yajima K, et al. & 2016 & 1 & 72 & $1 / 0$ & $0 / 1$ & $1 / 0$ & JI & 7 & 395 & 40 & - & - & - & 10 \\
\hline
\end{tabular}

$M$ male, $F$ female, $B$ benign, $M$ malignant, $O$ open, $L$ laparoscopy, $C v$ conversion, $C$ - $D I I I>$ clavien dindo classification III $>$

$L N$ lymph node, $B I$ BillrothI, BII BillrothII, RY Roux Y, GJ gastrojejunostomy, JI jejunal interposition 
Table $6 \quad$ Summary of Comparative Studies of LCTG for GSC

\begin{tabular}{|c|c|c|c|c|c|c|c|c|c|c|c|c|}
\hline Author & Year & $\begin{array}{l}\text { LCTG } \\
\text { OCTG }\end{array}$ & $\mathrm{n}$ & $\begin{array}{c}\text { initial } \\
\text { disease } \\
\text { (B / M) }\end{array}$ & $\begin{array}{c}\text { Previous } \\
\text { ope } \\
\text { Open/ Lap }\end{array}$ & $\begin{array}{l}\text { Operation } \\
\text { time } \\
\text { (min) }\end{array}$ & $\begin{array}{l}\text { Blood loss } \\
(\mathrm{ml})\end{array}$ & $\begin{array}{l}\text { open } \\
\text { CV } \\
(\%)\end{array}$ & $\begin{array}{c}\text { Morbidity } \\
(\mathrm{C}-\mathrm{D} \text { III }>) \\
(\%)\end{array}$ & $\begin{array}{c}\text { Retrieved } \\
\text { LN } \\
\text { (n) }\end{array}$ & $\begin{array}{c}\text { Hospital } \\
\text { stay } \\
\text { (days) }\end{array}$ & OS \\
\hline \multirow[t]{2}{*}{ Nagai E, et al. } & \multirow[t]{2}{*}{2014} & Lap & 12 & $7 / 5$ & $12 / 0$ & $362.3 \pm 68.4^{*}$ & $65.8 \pm 62.0$ & 0 & 0 & $23.7 \pm 10.7$ & $11.3 \pm 2.8^{*}$ & $\begin{array}{c}77.8 \% \\
(3 \mathrm{yr})\end{array}$ \\
\hline & & Open & 10 & $2 / 8$ & $10 / 0$ & $270.5 \pm 94.9$ & $746.3 \pm 577.1^{*}$ & - & $2(20)$ & $15.9 \pm 7.6$ & $24.9 \pm 10$ & $100 \%$ \\
\hline \multirow[t]{2}{*}{ Kwon IG, et al. } & \multirow[t]{2}{*}{2014} & Lap & 18 & $2 / 16$ & $11 / 7^{*}$ & $266.2 \pm 77.2^{*}$ & $182.2 \pm 188.7$ & $1(5.6)$ & $3(16.7)$ & $8(0-37)$ & $6(5-44)^{*}$ & $\begin{array}{c}94.9 \% \\
(5 \mathrm{vr})\end{array}$ \\
\hline & & Open & 58 & $12 / 46$ & $54 / 4$ & $203.3 \pm 52.2$ & $193.1 \pm 227.6$ & - & $9(15.5)$ & $7(0-36)$ & $9(6-28)$ & $100 \%$ \\
\hline \multirow[t]{2}{*}{ Kim HS, et al. } & \multirow[t]{2}{*}{2014} & Lap & 17 & $0 / 17$ & $10 / 7$ & $197.2 \pm 60.6^{*}$ & & 0 & $4(23.5)$ & $12.9 \pm 8.7$ & $11.1 \pm 8.7$ & \\
\hline & & Open & 50 & $0 / 50$ & & $149.3 \pm 46.9$ & & - & $15(30)$ & & $13.8 \pm 9.4$ & \\
\hline \multirow[t]{2}{*}{ Son SY, et al. } & \multirow[t]{2}{*}{2015} & Lap & 17 & $7 / 10$ & $11 / 6^{*}$ & $234.4 \pm 65.2^{*}$ & $227.6 \pm 245.0$ & $8(47.1)$ & $1(5.9)$ & $18.8 \pm 12.3$ & $9.3 \pm 3.2$ & $\begin{array}{c}66.7 \% \\
(5 \mathrm{yr})\end{array}$ \\
\hline & & Open & 17 & $6 / 11$ & $17 / 0$ & $170.0 \pm 39.5$ & $184.1 \pm 123.1$ & - & $1(5.9)$ & $22.3 \pm 14.4$ & $9.3 \pm 3.1$ & $60.3 \%$ \\
\hline \multirow[t]{2}{*}{ Tsunoda S, et al. } & \multirow[t]{2}{*}{2016} & Lap & 10 & $4 / 6$ & $10 / 0$ & $324.5 \pm 40.6$ & $55.4 \pm 60.7$ & 0 & 0 & $22.4 \pm 15.0$ & $12.5 \pm 2.7$ & \\
\hline & & Open & 6 & & & 289 & 893 & - & $2(33)$ & 7 & 24 & \\
\hline \multirow[t]{2}{*}{ Nakaji Y, et al. } & \multirow[t]{2}{*}{2019} & Lap & 4 & $6 / 16$ & & $455 *$ & 158 & 0 & $2(50.0)$ & 15 & 15 & \multirow{2}{*}{$\begin{array}{l}94 \% \\
(5 \mathrm{yr})\end{array}$} \\
\hline & & Open & 18 & & & 293 & 625 & - & $5(27.8)$ & 11 & 16 & \\
\hline \multirow[t]{2}{*}{ Booka E, et al. } & \multirow[t]{2}{*}{2019} & Lap & 8 & $4 / 4$ & $5 / 3$ & $307.5 \pm 56.0$ & $135.5 \pm 181.2^{*}$ & $2(25)$ & $3(37.5)$ & $8.8 \pm 4.6$ & $10.6 \pm 3.7$ & \\
\hline & & Open & 23 & $6 / 17$ & $21 / 2$ & $295.8 \pm 81.7$ & $568.3 \pm 446.4$ & - & $6(26.1)$ & $6.0 \pm 6.9$ & $21.3 \pm 37.3$ & \\
\hline \multirow[t]{2}{*}{ Otsuka R, et al. } & \multirow[t]{2}{*}{2019} & Lap & 7 & $5 / 2$ & $6 / 1$ & $364 \pm 95$ & $70 \pm 71 *$ & 0 & $2(28.6 \%)$ & $22 \pm 13$ & $13 \pm 5$ & \\
\hline & & Open & 20 & $11 / 9$ & $18 / 2$ & $309 \pm 104$ & $1066 \pm 1428$ & - & $10(50 \%)$ & $12 \pm 9$ & $27 \pm 21$ & \\
\hline \multirow[t]{2}{*}{ our data } & \multirow[t]{2}{*}{2020} & Lap & 17 & $5 / 12$ & $11 / 6$ & $242.6 \pm 64.8$ & $24.4 \pm 27.0$ * & 0 & 0 & $14.8 \pm 11.9$ & $14.8 \pm 5.1$ & $\begin{array}{l}88.2 \% \\
(5 \mathrm{yr})^{*}\end{array}$ \\
\hline & & Open & 20 & $4 / 16$ & $17 / 3$ & $202.5 \pm 67.6$ & $212.5 \pm 230.8$ & - & $2(10.0)$ & $12.0 \pm 10.4$ & $29.1 \pm 56.3$ & $55.0 \%$ \\
\hline
\end{tabular}

$L C T G$ laparoscopic completion total gastrectomy, GSC gastric stump cancer, $O C T G$ open completion total gastrectomy, $B$ benign disease, $M$ malignant disease, Lap laparoscopy, $C-D I I I>$ clavien dindo classification III $>, L N$ lymph node, $O S$ overall survival, $* P$ value $<0.05$

\section{Discussion}

Our results confirm the feasibility of LCTG for the treatment of GSC. Patients in the LCTG group had significantly longer operation times but significantly less blood loss and earlier flatus passage than the OCTG group. Furthermore, no conversion to open surgery and no higher morbidity than Clavien Dindo class III were reported in the LCTG group.

Yamada et al. [24] were the first to present a case report for laparoscopy-assisted resection of gastric remnant cancer in 2005; many additional studies reported successful applications of the technique [25-33]. Therefore, we searched reports of LCTG for GSC from the PubMed database in English literature and summarized the data by case reports (Table 5) and comparative studies (Table 6). Many surgeons have successfully applied LCTG, and this technique is considered the preferred treatment option (Table 5) [24-33]. As shown in Table 6, our results are consistent with those of comparative studies [14-21]. 
Table 5

Summary of Case Reports of LCTG for GSC

\begin{tabular}{|c|c|c|c|c|c|c|c|c|c|c|}
\hline $\begin{array}{l}\text { Hospital } \\
\text { stay }\end{array}$ & (days) & - & $\begin{array}{l}11(8- \\
18)\end{array}$ & - & - & $8.8(8-9)$ & $\begin{array}{l}8(7- \\
9)\end{array}$ & 13 & $\begin{array}{l}18(13- \\
26)\end{array}$ & 7 \\
\hline $\begin{array}{l}\text { Retrieved } \\
\text { LN }\end{array}$ & (n) & - & $\begin{array}{l}18(12- \\
26)\end{array}$ & $\begin{array}{l}14.5 \\
(9- \\
20)\end{array}$ & $18 \pm 5$ & $\begin{array}{l}18.2(12- \\
24)\end{array}$ & $\begin{array}{l}16.7 \\
(10- \\
22)\end{array}$ & 24 & - & 20 \\
\hline $\begin{array}{l}\text { Morbidity } \\
(C-D \text { III>) }\end{array}$ & (n) & - & $\begin{array}{l}\text { fistula } \\
\text { (1) }\end{array}$ & - & SSI (1) & - & - & - & $\begin{array}{l}\text { bleeding } \\
\text { (1) }\end{array}$ & - \\
\hline $\begin{array}{l}\text { Open } \\
\text { conversion }\end{array}$ & (n) & - & - & - & 1 & - & - & - & - & - \\
\hline Blood loss & $(\mathrm{ml})$ & 30 & $\begin{array}{l}19(0- \\
264)\end{array}$ & $\begin{array}{l}425 \\
(400- \\
450)\end{array}$ & $\begin{array}{l}110 \pm \\
40 \mathrm{ml}\end{array}$ & $\begin{array}{l}63.6(10- \\
233)\end{array}$ & $\begin{array}{l}76.7 \\
(50- \\
100)\end{array}$ & 100 & $\begin{array}{l}270.3 \\
(33- \\
500)\end{array}$ & 200 \\
\hline $\begin{array}{l}\text { Operation } \\
\text { time }\end{array}$ & $(\min )$ & 274 & $\begin{array}{l}210 \\
(160- \\
260)\end{array}$ & $\begin{array}{l}487.5 \\
(435- \\
540)\end{array}$ & $\begin{array}{l}205 \pm \\
25 \mathrm{~min}\end{array}$ & $\begin{array}{l}370.8 \\
(258-540)\end{array}$ & $\begin{array}{l}251.7 \\
(225- \\
280)\end{array}$ & 200 & $\begin{array}{l}413.3 \\
(367- \\
488)\end{array}$ & 295 \\
\hline interval & (yrs) & 10 & 15 & 1.5 & - & - & $\begin{array}{l}22 \\
(6- \\
30)\end{array}$ & 28 & $\begin{array}{l}19(0.08- \\
50)\end{array}$ & 25 \\
\hline $\begin{array}{l}\text { Previous } \\
\text { reconstruction }\end{array}$ & & BII & BII (3) & BII (2) & $\begin{array}{l}\mathrm{BI}(2) / \\
\mathrm{BII} \\
(13)\end{array}$ & $\begin{array}{l}\mathrm{BI}(3) / \mathrm{BII} \\
(2)\end{array}$ & $\begin{array}{l}\mathrm{BII}(2) \\
/ \mathrm{RY} \\
(1)\end{array}$ & BII & $\begin{array}{l}\mathrm{BI}(2) / \\
\mathrm{BII}(1) / \\
\operatorname{RY}(1)\end{array}$ & $\begin{array}{l}\mathrm{GJ} \\
\text { with } \\
\text { braun }\end{array}$ \\
\hline $\begin{array}{l}\text { previous } \\
\text { ope }\end{array}$ & $\begin{array}{l}\text { (Open } \\
\text { / Lap) }\end{array}$ & $1 / 0$ & $3 / 0$ & $1 / 1$ & - & - & $3 / 0$ & $1 / 0$ & $3 / 1$ & $1 / 0$ \\
\hline $\begin{array}{l}\text { initial } \\
\text { disease }\end{array}$ & $\begin{array}{l}(\mathrm{B} / \\
\mathrm{M})\end{array}$ & $0 / 1$ & $3 / 0$ & $0 / 1$ & - & $1 / 4$ & $2 / 1$ & $0 / 1$ & $1 / 3$ & $0 / 1 *$ \\
\hline gender & $\begin{array}{l}(\mathrm{M} / \\
\mathrm{F})\end{array}$ & $1 / 0$ & $3 / 0$ & $1 / 1$ & $13 / 2$ & $3 / 2$ & $3 / 0$ & $0 / 1$ & $4 / 0$ & $1 / 0$ \\
\hline age & & 69 & $59-73$ & $\begin{array}{l}37 / \\
68\end{array}$ & $\begin{array}{l}53.5 \\
(40- \\
76)\end{array}$ & $\begin{array}{l}54-84 \\
(70.4)\end{array}$ & $\begin{array}{l}68.7 \\
(55- \\
76)\end{array}$ & 83 & $\begin{array}{l}76.5 \\
(62-83)\end{array}$ & 73 \\
\hline$n$ & & 1 & 3 & 2 & 15 & 5 & 3 & 1 & 4 & 1 \\
\hline Year & & 2005 & 2008 & 2009 & 2010 & 2013 & 2014 & 2015 & 2015 & 2016 \\
\hline Author & & $\begin{array}{l}\text { Yamada. } \\
\mathrm{H} \text {, et al. }\end{array}$ & $\begin{array}{l}\text { Corcione. } \\
\text { F, et al. }\end{array}$ & $\begin{array}{l}\text { Cho. } \\
\text { HJ, et } \\
\text { al. }\end{array}$ & $\begin{array}{l}\text { Qian F, } \\
\text { et al. }\end{array}$ & $\begin{array}{l}\text { Shinohara. } \\
\text { T, et al. }\end{array}$ & $\begin{array}{l}\text { Pan } \\
\text { et al. }\end{array}$ & $\begin{array}{l}\text { Kim } \\
\text { HI, et } \\
\text { al. }\end{array}$ & $\begin{array}{l}\text { Korehisa } \\
\text { S, et al. }\end{array}$ & $\begin{array}{l}\text { Kim } \\
\text { DJ, et } \\
\text { al. }\end{array}$ \\
\hline
\end{tabular}

$M$ male, $F$ female, $B$ benign, $M$ malignant, Lap laparoscopy, $L N$ lymph node, $C-D$ III >clavien dindo classification III>, BIBillrothl, $B / /$ Billrothll, $R Y$ Roux-en Y, GJ gastrojejunostomy, * common bile duct cancer 
Table 6

Summary of Comparative Studies of LCTG for GSC

\begin{tabular}{|c|c|c|c|c|c|c|c|c|c|c|c|c|}
\hline OS & & $\begin{array}{l}77.8 \% \\
\text { (3 year) }\end{array}$ & $100 \%$ & $\begin{array}{l}94.9 \% \\
\text { (5 year) }\end{array}$ & $100 \%$ & & & $\begin{array}{l}66.7 \% \\
\text { (5 year) }\end{array}$ & $60.30 \%$ & & & $\begin{array}{l}94 \% \\
\text { (5 year) }\end{array}$ \\
\hline $\begin{array}{l}\text { Hospital } \\
\text { stay }\end{array}$ & (days) & $\begin{array}{l}11.3 \pm \\
2.8 *\end{array}$ & $\begin{array}{l}24.9 \pm \\
10\end{array}$ & $\begin{array}{l}6(5- \\
44) *\end{array}$ & $\begin{array}{l}9(6- \\
28)\end{array}$ & $\begin{array}{l}11.1 \\
\pm 8.7\end{array}$ & $\begin{array}{l}13.8 \\
\pm 9.4\end{array}$ & $\begin{array}{l}9.3 \pm \\
3.2\end{array}$ & $\begin{array}{l}9.3 \pm \\
3.1\end{array}$ & $\begin{array}{l}12.5 \pm \\
2.7\end{array}$ & 24 & 15 \\
\hline $\begin{array}{l}\text { Retrieved } \\
\text { LN }\end{array}$ & (n) & $\begin{array}{l}23.7 \pm \\
10.7\end{array}$ & $\begin{array}{l}15.9 \pm \\
7.6\end{array}$ & $\begin{array}{l}8(0- \\
37)\end{array}$ & $\begin{array}{l}7(0- \\
36)\end{array}$ & $\begin{array}{l}12.9 \\
\pm 8.7\end{array}$ & & $\begin{array}{l}18.8 \pm \\
12.3\end{array}$ & $\begin{array}{l}22.3 \pm \\
14.4\end{array}$ & $\begin{array}{l}22.4 \pm \\
15.0\end{array}$ & 7 & 15 \\
\hline $\begin{array}{l}\text { Morbidity } \\
\text { (C-D III>) }\end{array}$ & (\%) & 0 & $2(20)$ & $\begin{array}{l}3 \\
(16.7)\end{array}$ & $\begin{array}{l}9 \\
(15.5)\end{array}$ & $\begin{array}{l}4 \\
(23.5)\end{array}$ & $\begin{array}{l}15 \\
(30)\end{array}$ & $1(5.9)$ & $1(5.9)$ & 0 & 2(33) & $2(50)$ \\
\hline $\begin{array}{l}\text { Open } \\
\text { conversion }\end{array}$ & (\%) & 0 & - & $1(5.6)$ & - & 0 & - & $\begin{array}{l}8 \\
(47.1)\end{array}$ & - & 0 & - & 0 \\
\hline Blood loss & $(\mathrm{ml})$ & $\begin{array}{l}65.8 \pm \\
62.0\end{array}$ & $\begin{array}{l}746.3 \\
\pm \\
577.1^{*}\end{array}$ & $\begin{array}{l}182.2 \\
\pm 188.7\end{array}$ & $\begin{array}{l}193.1 \\
\pm \\
227.6\end{array}$ & & & $\begin{array}{l}227.6 \\
\pm 245.0\end{array}$ & $\begin{array}{l}184.1 \\
\pm 123.1\end{array}$ & $\begin{array}{l}55.4 \pm \\
60.7\end{array}$ & 893 & 158 \\
\hline $\begin{array}{l}\text { Operation } \\
\text { time }\end{array}$ & $(\min )$ & $\begin{array}{l}362.3 \\
\pm 68.4 \text { * }\end{array}$ & $\begin{array}{l}270.5 \\
\pm 94.9\end{array}$ & $\begin{array}{l}266.2 \\
\pm 77.2^{\star}\end{array}$ & $\begin{array}{l}203.3 \\
\pm 52.2\end{array}$ & $\begin{array}{l}197.2 \\
\pm \\
60.6^{\star}\end{array}$ & $\begin{array}{l}149.3 \\
\pm 46.9\end{array}$ & $\begin{array}{l}234.4 \\
\pm 65.2^{*}\end{array}$ & $\begin{array}{l}170.0 \\
\pm 39.5\end{array}$ & $\begin{array}{l}324.5 \pm \\
40.6\end{array}$ & 289 & 455 * \\
\hline $\begin{array}{l}\text { previous } \\
\text { ope }\end{array}$ & $\begin{array}{l}\text { (Open } \\
\text { / Lap) }\end{array}$ & $12 / 0$ & $10 / 0$ & $11 / 7^{\star}$ & $54 / 4$ & $10 / 7$ & & $11 / 6^{*}$ & $17 / 0$ & $10 / 0$ & & \\
\hline $\begin{array}{l}\text { initial } \\
\text { disease }\end{array}$ & $\begin{array}{l}(\mathrm{B} / \\
\mathrm{M})\end{array}$ & $7 / 5$ & $2 / 8$ & $2 / 16$ & $\begin{array}{l}12 / \\
46\end{array}$ & $0 / 17$ & $0 / 50$ & $7 / 10$ & $6 / 11$ & $4 / 6$ & & $6 / 16$ \\
\hline$n$ & & 12 & 10 & 18 & 58 & 17 & 50 & 17 & 17 & 10 & 6 & 4 \\
\hline LCTG & OCTG & Lap & Open & Lap & Open & Lap & Open & Lap & Open & Lap & Open & Lap \\
\hline Year & & 2014 & & 2014 & & 2014 & & 2015 & & 2016 & & 2019 \\
\hline Author & & $\begin{array}{l}\text { Nagai } \\
\text { E, et al. }\end{array}$ & & $\begin{array}{l}\text { Kwon } \\
\text { IG, et } \\
\text { al. }\end{array}$ & & $\begin{array}{l}\text { Kim } \\
\text { HS, et } \\
\text { al. }\end{array}$ & & $\begin{array}{l}\text { Son SY, } \\
\text { et al. }\end{array}$ & & $\begin{array}{l}\text { Tsunoda } \\
\text { S, et al. }\end{array}$ & & $\begin{array}{l}\text { Nakaji } \\
\text { Y, et al. }\end{array}$ \\
\hline
\end{tabular}

LCTG laparoscopic completion total gastrectomy, GSC gastric stump cancer, OCTG open completion total gastrectomy, $B$ benign disease, $M$ malignant diseas overall survival, * $P$ value $<0.05$

The patients in the LCTG group had smaller tumor size, lower numbers of metastatic lymph nodes, and lower pathological stages than patients in the OCTG group; however, the clinical stage distribution, dissection, and retrieved lymph nodes showed no significant differences between the groups. Additionally, the number of retrieved lymph nodes was equal to that reported in other studies (Table 6) [14-21]. The OCTG group had a higher number of recurrences and deaths than the LCTG group, because the OCTG group had more advanced cases, leading to a worse 5-year overall survival rate than the LCTG group. Recently, the number of lower stages of GSC has been increasing due to the strict postoperative surveillance for PGC; this surveillance combined with the feasibility and validity of LCTG can improve patient survival [7].

The most difficult part of the operative procedure for GSC is the adhesiolysis, which is the key factor to safely performing LCTG [18]. A precise and sharp dissection between the adjacent organ and remnant stomach is necessary to avoid organ injury, and less bowel manipulation leads to early recovery [15, 18]. We consider laparoscopy an effective solution to overcome this difficulty in the treatment of GSC. The advantages of laparoscopic surgery are pneumoperitoneum, which widens the dissectible layer between the adhered organs, and a magnified view that enables detection of the loose and dissectible layer. Moreover, progressive high definition (HD) imaging significantly contributes to the benefits from such magnified views. We have been using the HD scope system (Karl Storz SE \& Co. KG, Tuttlingen, DE) since the introduction of LCTG in our institution. Advanced energy devices and forceps also contribute to refining the quality of surgery, reducing bleeding, reducing the trauma to organs, and refining the precision of lymphadenectomy. Our sophisticated dissection techniques combined with these advanced developments enable us to perform LCTG with an extremely reduced blood loss compared to previous casecontrolled studies (Table 6), leading to earlier recovery of digestive peristalsis.

Robotic gastrectomy could be a future advancement for the treatment of GSC in terms of its visual improvement in the surgical field, which is referred to as robotically enhanced surgical anatomy [34]. The refined anatomical view of robotic gastrectomy could achieve precise movement of forceps without hand tremors, which could increase operative accuracy. In fact, robotic gastrectomy for PGC has decreased the complication rate despite longer operative time and higher cost than laparoscopic gastrectomy $[35,36]$. Robotic surgery has already been applied to GSC and reviewed retrospectively, which has shown a lower conversion rate and comparative short-term outcomes to LCTG [37]. We have also introduced robotic surgery to GSC and expect superior results.

We acknowledge some limitations in our study. First, this study had a retrospective design, which could have led to potential selection biases. Therefore, a randomized, controlled study should be completed. Second, due to the low incidence of GSC, the sample size was too small to elucidate the universal results and superiority of LCTG over OCTG for GSC. A multicenter study is necessary to validate our results.

\section{Conclusion}


LCTG was safely conducted without complications and mortality, implicating the oncological validity of LCTG for the treatment of GSC. With sophistication of laparoscopic skills and advanced technologies, LCTG is less invasive, results in reduced blood loss, and leads to earlier recovery of intestinal peristalsis.

\section{Abbreviations}

GSC

Gastric stump cancer; PGC:Primary gastric cancer; LCTG:Laparoscopic completion total gastrectomy; OCTG:Open completion total gastrectomy; HD:High definition

\section{Declarations}

\section{Ethics approvaland consent to participate}

All procedures performed in this study that involved human participants were in accordance with the ethical standards of the institutional and/or national research committee and the 1964 Helsinki declaration and its later amendments or comparable ethical standards. This study was reviewed and approved by the Ethics Committee of Ishikawa Prefectural Central Hospital. Reference number: 1438. Informed consent was obtained from all individual participants included in the study.

\section{Consent for publication}

Not applicable

\section{Availability of data and materials}

All data and materials are contained within the manuscript.

\section{Competing interests}

The authors declare that they have no competing interests.

\section{Funding}

The authors have no financial ties to disclose.

\section{Authors' contributions}

Study conception and design: ToTa, NI. Analysis and interpretation of data: ToTa, NI, ToTs SK. Drafting of manuscript: ToTa, DY, HK. Acquisition of data: ToTa, HS, YS, KH. Critical revision of manuscript: ToTa, NI, SK, HB. All authors have read and approved the final manuscript.

\section{Acknowledgements}

We would like to thank Editage (www.editage.com) for English language editing.

\section{References}

1. Kaneko K, Kondo H, Saito D, Shirao K, Yamaguchi H, Yokota T, et al. Early gastric stump cancer following distal gastrectomy. Gut. 1998;43:342-4.

2. Sinning C, Schaefer N, Standop J, Hirner A, Wolff M. Gastric stump carcinoma - epidemiology and current concepts in pathogenesis and treatment. Eur J Surg Oncol. 2007;33:133-9.

3. Schrumpf E, Serck-Hanssen A, Stadaas J, Aune S, Myren J, Osnes M. Mucosal changes in the gastric stump 20-25 years after partial gastrectomy. Lancet. 1977;2:467-9.

4. Ohashi M, Katai H, Fukagawa T, Gotoda T, Sano T, Sasako M. Cancer of the gastric stump following distal gastrectomy for cancer. Br J Surg. 2007;94:925.

5. Di Leo A, Pedrazzani C, Bencivenga M, Coniglio A, Rosa F, Morgani P, et al. Gastric stump cancer after distal gastrectomy for benign disease: clinicopathological features and surgical outcomes. Ann Surg Oncol. 2014;21:2594-600.

6. Morgagni P, Gardini A, Marrelli D, Vittimberga G, Marchet A, de Manzoni G, et al. Gastric stump carcinoma after distal subtotal gastrectomy for early gastric cancer: experience of 541 patients with long-term follow-up. Am J Surg. 2015;209:1063-8.

7. Takahashi M, Takeuchi H, Tsuwano S, Nakamura R, Takahashi T, Wada N, et al. Surgical Resection of Remnant Gastric Cancer Following Distal Gastrectomy: A Retrospective Clinicopathological Study. Ann Surg Oncol. 2016;23:511-21.

8. Ahn HS, Kim JW, Yoo MW, Park DJ, Lee HJ, Lee KU, et al. Clinicopathological features and surgical outcomes of patients with remnant gastric cancer after a distal gastrectomy. Ann Surg Oncol. 2008;15:1632-9.

9. Ohira M. Current status in remnant gastric cancer after distal gastrectomy. World J Gastroenterol. 2016;22:2424.

10. Tokunaga M, Sano T, Ohyama S, Hiki N, Fukunaga T, Yamada K, et al. Clinicopathological characteristics and survival difference between gastric stump carcinoma and primary upper third gastric cancer. J Gastrointest Surg. 2013;17:313-8. 
11. Hosono S, Arimoto Y, Ohtani H, Kanamiya Y. Meta-analysis of short-term outcomes after laparoscopy-assisted distal gastrectomy. World J Gastroenterol. 2006;12:7676-83.

12. Katai H, Sasako M, Fukuda H, Nakamura K, Hiki N, Saka M, et al. Safety and feasibility of laparoscopy-assisted distal gastrectomy with suprapancreatic nodal dissection for clinical stage I gastric cancer: a multicenter phase II trial (JCOG 0703). Gastric Cancer. 2010;13:238-44.

13. Kim HH, Han SU, Kim MC, Kim W, Lee HJ, Ryu SW, et al. Effect of Laparoscopic Distal Gastrectomy vs Open Distal Gastrectomy on Long-term Survival Among Patients With Stage I Gastric Cancer: The KLASS-01 Randomized Clinical Trial. JAMA Oncol. 2019;5:506-13.

14. Nagai E, Nakata K, Ohuchida K, Miyasaka Y, Shimizu S, Tanaka M. Laparoscopic total gastrectomy for remnant gastric cancer: feasibility study. Surg Endosc. 2014;28:289-96.

15. Kwon IG, Cho I, Guner A, Choi YY, Shin HB, Kim HI, et al. Minimally invasive surgery for remnant gastric cancer: a comparison with open surgery. Surg Endosc. 2014;28:2452-8.

16. Kim HS, Kim BS, Lee IS, Lee S, Yook JH, Kim BS. Laparoscopic gastrectomy in patients with previous gastrectomy for gastric cancer: a report of 17 cases. Surg Laparosc Endosc Percutan Tech. 2014;24:177-82.

17. Son SY, Lee CM, Jung DH, Lee JH, Ahn SH, Park DJ, et al. Laparoscopic completion total gastrectomy for remnant gastric cancer: a single-institution experience. Gastric Cancer. 2015;18:177-82.

18. Tsunoda S, Okabe H, Tanaka E, Hisamori S, Harigai M, Murakami K, et al. Laparoscopic gastrectomy for remnant gastric cancer: a comprehensive review and case series. Gastric Cancer. 2016;19:287-92.

19. Nakaji YU, Saeki H, Kudou K, Nakanishi R, Sugiyama M, Nakashima Y, et al. Short-and Long-term Outcomes of Surgical Treatment for Remnant Gastric Cancer After Distal Gastrectomy. Anticancer Res. 2019;39:1411-5.

20. Booka E, Kaihara M, Mihara K, Nishiya S, Handa K, Ito Y, et al. Laparoscopic total gastrectomy for remnant gastric cancer: A single-institution experience. Asian J Endosc Surg. 2019;12:58-63.

21. Otsuka R, Hayashi H, Sakata H, Uesato M, Hayano K, Murakami K, et al. Short-term clinical outcomes of laparoscopic gastrectomy for remnant gastric cancer: A single-institution experience and systematic review of the literature. Ann Gastroenterol Surg. 2019;3:181-6.

22. Inaki N. Laparoscopic distal gastrectomy and Roux-en-Y reconstruction. Ann Laparosc Endosc. 2017;2.

23. Japanese Gastric Cancer A. Japanese gastric cancer treatment guidelines 2014 (ver. 4). Gastric Cancer. 2017;20:1-19.

24. Yamada H, Kojima K, Yamashita T, Kawano T, Sugihara K, Nihei Z. Laparoscopy-assisted resection of gastric remnant cancer. Surg Laparosc Endosc Percutan Tech. 2005;15:226-9.

25. Corcione F, Pirozzi F, Marzano E, Cuccurullo D, Settembre A, Miranda L. Laparoscopic approach to gastric remnant-stump: our initial successful experience on 3 cases. Surg Laparosc Endosc Percutan Tech. 2008;18:502-5.

26. Cho HJ, Kim W, Hur H, Jeon HM. Laparoscopy-assisted completion total gastrectomy for gastric cancer in remnant stomach: report of 2 cases. Surg Laparosc Endosc Percutan Tech. 2009;19:e57-60.

27. Qian F, Yu PW, Hao YX, Sun G, Tang B, Shi Y, et al. Laparoscopy-assisted resection for gastric stump cancer and gastric stump recurrent cancer: a report of 15 cases. Surg Endosc. 2010;24:3205-9.

28. Shinohara T, Hanyu N, Tanaka Y, Murakami K, Watanabe A, Yanaga K. Totally laparoscopic complete resection of the remnant stomach for gastric cancer. Langenbecks Arch Surg. 2013;398:341-5.

29. Pan Y, Mou YP, Chen K, Xu XW, Cai JQ, Wu D, et al. Three cases of laparoscopic total gastrectomy with intracorporeal esophagojejunostomy for gastric cancer in remnant stomach. World J Surg Oncol. 2014;12:342.

30. Kim HI, Kim MG. Entirely Laparoscopic Gastrectomy and Colectomy for Remnant Gastric Cancer with Gastric Outlet Obstruction and Transverse Colon Invasion. J Gastric Cancer. 2015;15:286-9.

31. Korehisa S, Ohgaki K, Yukaya T, Zaitu Y, Tsuda Y, Kasagi Y, et al. Laparoscopic Total Gastrectomy for RGC: Four Case Reports. Anticancer Res. 2015;35:5023-6.

32. Kim DJ, Kim W. Laparoscopic completion total gastrectomy for remnant gastric cancer following pancreaticoduodenectomy for bile duct cancer: a case report. Ann Surg Treat Res. 2016;90:106-10.

33. Yajima K, Iwasaki Y, Yuu K, Oohinata R, Amaki M, Kohira Y, et al. A Case of Laparoscopic Resection for Carcinoma of the Gastric Remnant following Proximal Gastrectomy Reconstructed with Jejunal Interposition. Case Rep Surg. 2016. doi:10.1155/2016/9357659.

34. Noshiro H, Ikeda O, Urata M. Robotically-enhanced surgical anatomy enables surgeons to perform distal gastrectomy for gastric cancer using electric cautery devices alone. Surg Endosc. 2014;28:1180-7.

35. Kim HI, Han SU, Yang HK, Kim YW, Lee HJ, Ryu KW, et al. Multicenter Prospective Comparative Study of Robotic Versus Laparoscopic Gastrectomy for Gastric Adenocarcinoma. Ann Surg. 2016;263:103-9.

36. Uyama I, Suda K, Nakauchi M, Kinoshita T, Noshiro H, Takiguchi S, et al. Clinical advantages of robotic gastrectomy for clinical stage I/II gastric cancer: a multi-institutional prospective single-arm study. Gastric Cancer. 2019;22:377-85.

37. Alhossaini RM, Altamran AA, Cho M, Roh CK, Seo WJ, Choi S, et al. Lower rate of conversion using robotic-assisted surgery compared to laparoscopy in completion total gastrectomy for remnant gastric cancer. Surg Endosc. 2020;34:847-52.

\section{Figures}


Fig. 1

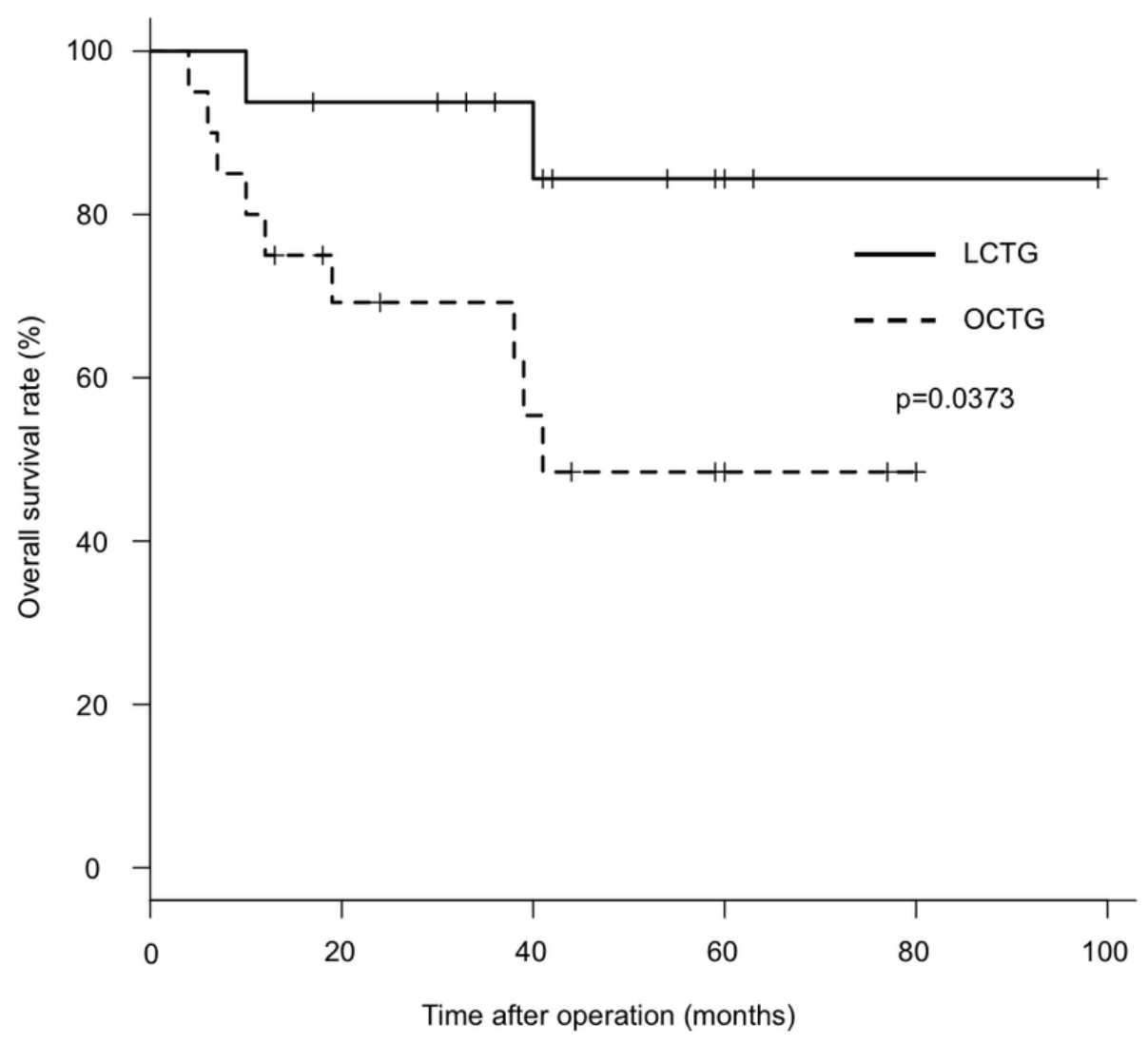

Figure 1

Overall survival rate Kaplan-Meier estimates of overall survival probability. The straight and dotted lines indicate the laparoscopic (LCTG) and open completion total gastrectomy (OCTG) groups, respectively. There was a significant difference between the two groups in the log-rank test $(p=0.0373)$ 
Fig. 2

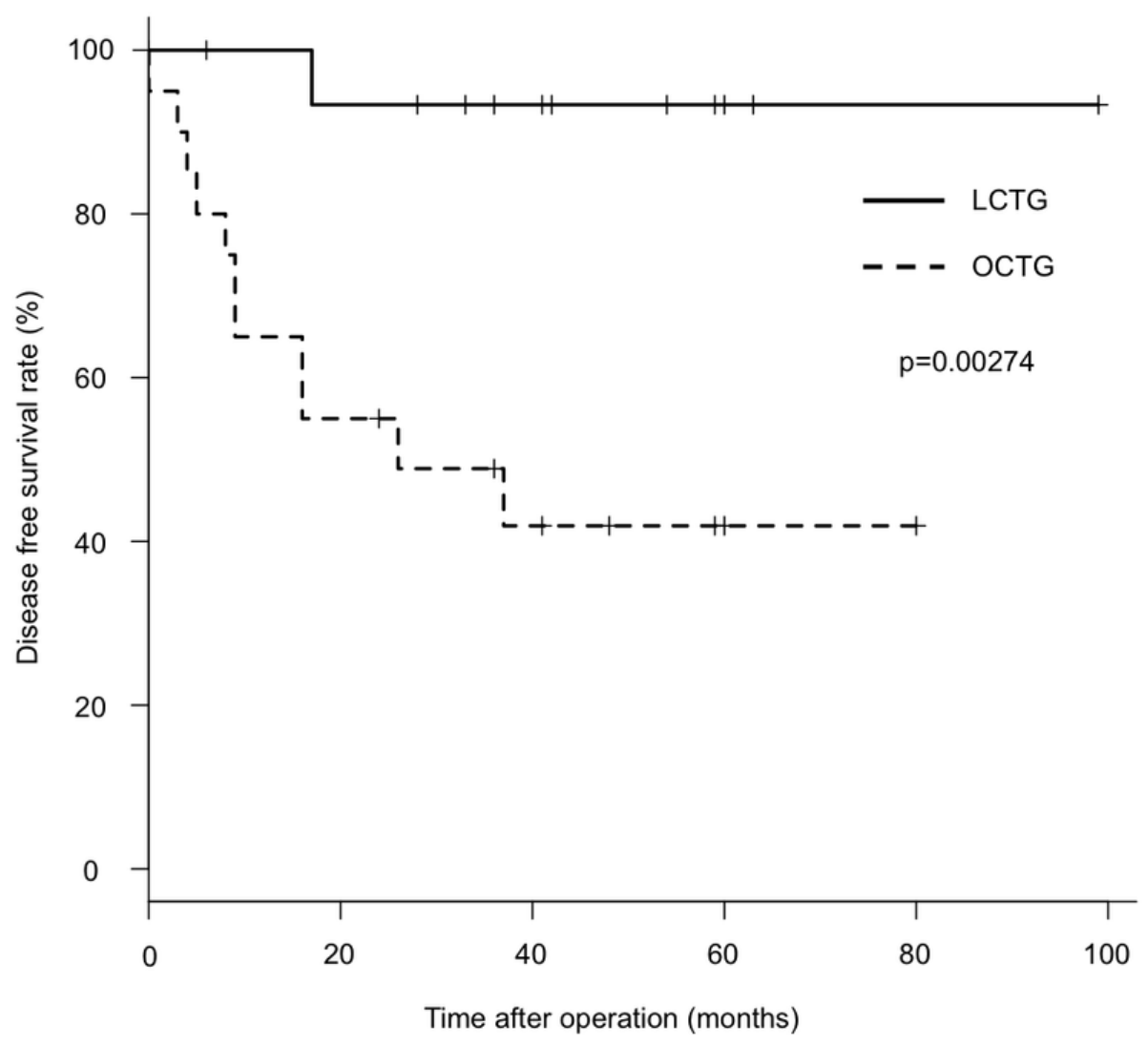

Figure 2

Disease-free survival rate Kaplan-Meier estimates of disease-free survival probability. The straight and dotted lines indicate the laparoscopic (LCTG) and open completion total gastrectomy (OCTG) groups, respectively. There was a significant difference between the two groups in the log-rank test $(p=0.00274)$ 